\title{
Platelet abnormalities induced by the administration of oestrogens
}

\author{
J. R. HAMPTON \\ From the Department of Medicine, Nottingham University
}

SYNOPSIS Platelet behaviour has been studied in a group of men and women treated with a variety of oestrogens. Although platelet aggregation is affected, changes in platelet electrophoretic behaviour are more striking. The administration of oral contraceptives containing oestrogens induces an abnormality in platelets and plasma lipids similar to that seen in patients with arterial disease.

The risk of arterial disease is lower in premenopausal women than in men of the same age (Kannell, Dawber, Kagan, Rivotskie, and Stokes, 1961), and it seems reasonable to suppose that this is related to an effect of female sex hormones. At first sight it is therefore surprising that three different groups of observations should suggest that the administration of oestrogens causes an increase in the risk of vascular disease. Contraceptive preparations containing oestrogens appear to lead to an increase in the incidence of venous (and possibly arterial) disease (Inman and Vessey, 1968; Vessey and Doll, 1968); the suppression of lactation with oestrogens appears to be associated with an increased incidence of venous disease (Daniel, Campbell, and Turnbull, 1967); and treatment of prostatic carcinoma with oestrogens seems to be associated with an increased mortality from cardiovascular disease (Veterans Administration, 1967).

Platelet masses form an essential part of a thrombus and the mechanism by which platelets stick to each other and to the vessel wall is therefore of extreme importance. Unfortunately this mechanism is not fully understood, largely because present techniques for studying the behaviour of human platelets are inadequate. All the techniques used to assess platelet function in vitro involve highly artificial situations and may have little relevance to the behaviour of platelets in vivo: when planning a study of the effects of oestrogens on human platelets we therefore decided to use three entirely different methods of following platelet function.

\section{Techniques}

Platelet adhesiveness was measured by a modification of Payling Wright's technique (Payling Wright, 1942). Platelet counts are made before and after rotation of citrated whole blood in a glass flask, and the difference between the two counts represents the platelets that have adhered to the glass.

Platelet aggregation was measured by the turbidimetric method of Born (1962). Light is passed through platelet-rich plasma; when aggregation is induced by such agents as adenosine diphosphate (ADP) or noradrenaline the optical density of the system falls and if the aggregates disperse it rises once more.

Platelet electrophoretic mobility was measured in a horizontal capillary apparatus using the technique described by Hampton and Mitchell (1966a). Incubation of platelets with ADP and noradrenaline induces a biphasic mobility response: low concentrations of these agents increase platelet mobility, and higher concentrations decrease it. No platelet aggregates form in this system because the platelets are in dilute suspension and the mixture is not stirred. It is not practicable to construct detailed mobility response 


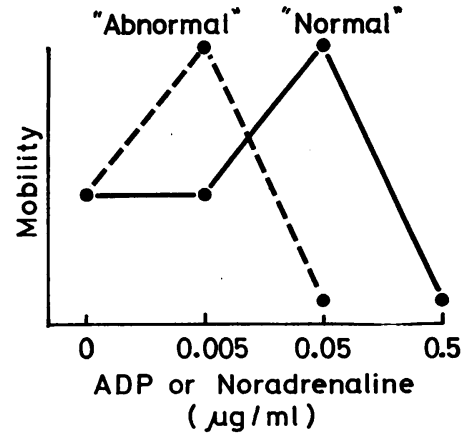

Fig. 1. Diagrammatic represention of normal and abnormal platelet electrophoretic mobility response to $A D P$ or noradrenaline.

curves, and usually only three or four points on the curve are determined, using $0.0005,0.005$, 0.05 , and $0.5 \mu \mathrm{g} / \mathrm{ml}$ of ADP or noradrenaline. The maximum possible platelet mobility is therefore not always recorded, but we have found that arbitrary definitions of 'normal' and 'abnormal' platelet behaviour are useful. In the normal response $0.05 \mu \mathrm{g} / \mathrm{ml}$ of ADP or noradrenaline induces a greater increase in mobility than 0.005 $\mu \mathrm{g} / \mathrm{ml}$; in the abnormal response 0.005 (or 0.0005 ) $\mu \mathrm{g} / \mathrm{ml}$ of either agent induces a greater increase in mobility than $0.05 \mu \mathrm{g} / \mathrm{ml}$ (Fig. 1).

We have previously described two abnormal patterns of platelet electrophoretic behaviour. Patients with any form of acute illness show abnormal platelet sensitivity to both ADP and noradrenaline (Hampton and Mitchell, 1966b); patients with arterial disease, on the other hand, show a selective increase in sensitivity to ADP alone (Hampton and Mitchell, 1966c). The abnormal behaviour of the platelets in patients with arterial disease appears to be related to an alteration in the plasma lipids, particularly to a change in the phospholipids of the low-density lipoproteins (Bolton, Hampton, and Mitchell, 1967).

\section{Experimental Design}

We studied the effects of synthetic and natural oestrogens on the platelets of a group of healthy males aged 22-32 years; the oestrogens used were ethinyl oestradiol $0.05 \mathrm{mg}$ daily, mestranol 0.1 and $0.02 \mathrm{mg}$ daily, and Premarin (conjugated equine oestrogen) $2 \cdot 5-10 \mathrm{mg}$ daily. Each compound was administered for a week in the first instance. The subjects were given active and placebo tablets sequentially, and neither they nor the investigators knew which they were receiving. The platelets of this group of subjects were studied by all three techniques, and the detailed results have been published elsewhere (Elkeles, Hampton, and Mitchell, 1968).

The effects of oral contraceptives were studied in a group of 12 healthy women aged 20 to 45 years. Only the electrophoretic technique was used, and 28 observations were made during $1 \overline{8}$ treatment cycles. The contraceptive preparations were of the combined oestrogen-progestoge variety in 10 subjects (Gynovlar 21, Ovulen; Norinyl 1, Orthonovin, and Anovlar) and of the sequential type in two (Sequens and Feminor). It a separate study a pure progestogen, chlormadEnone, was administered to three subjects. Fow convenience I shall refer to the day the first table is taken as day 1 ; days 22-28 are therefore the days on which no tablets are taken.

\section{Effect of Oestrogens in Men}

No statistically significant changes in plateleq adhesiveness were induced by any of thre oestrogens. Certain aspects of platelet aggregatio $\overrightarrow{n_{1}}$ were, however, enhanced during the period of

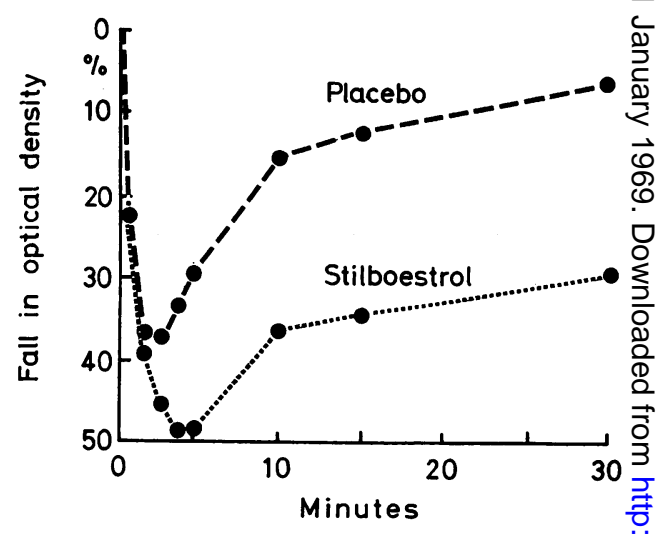

Fig. 2. Effect of stilboestrol on platelet aggregation induced by adenosine diphosphate $0.5 \mathrm{mg} / \mathrm{ml}(n=3)$. त

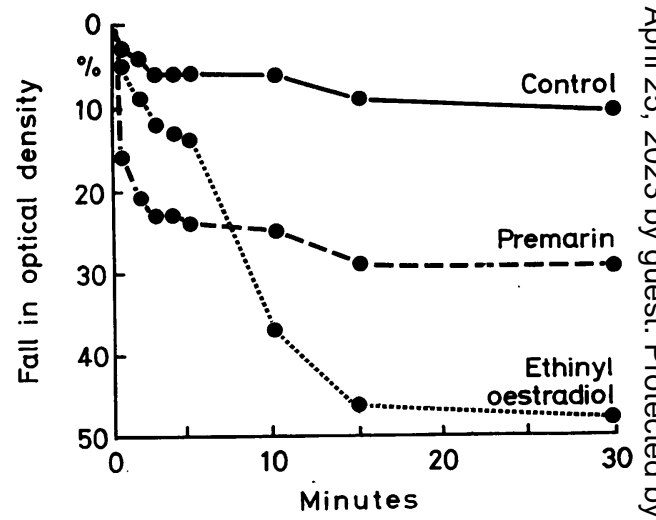

Fig. 3. Effect of Premarin and ethinyl oestradiol on 8 platelet aggregation induced by noradrenaline 0.5 $m g / m l(n=3)$. 


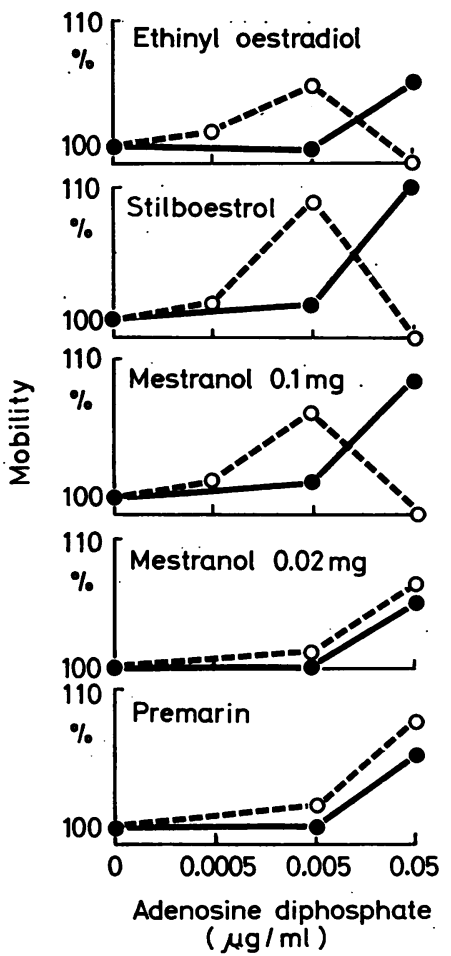

Fig. 4. Effect of oestrogens on changes in platelet electrophoretic mobility induced by adenosine diphosphate. (Ethinyl oestradiol, $n=3 ;$ Mestranol $0.1 \mathrm{mg}, \mathrm{n}=3$; Mestranol $0.02 \mathrm{mg}, n=2$; Premarin, $n=4$.) $($ Filled circles $=$ control; open circles $=$ oestrogen.)

treatment with the active tablets as opposed to the placebos. Figure 2 shows that during treatment with stilboestrol there was an increase in the maximum fall in optical density induced by ADP and that disaggregation was diminished. The effect of the other oestrogens was similar. All the oestrogens also increased the maximum aggregation induced by noradrenaline, and the effect of low concentrations of noradrenaline was particularly enhanced. Figure 3 shows the platelet aggregation pattern during treatment with ethinyl oestradiol and Premarin: these compounds were tested sequentially on the same group of subjects, and the results obtained in the control periods have been combined.

Observations made with the electrophoretic technique showed the oestrogens to have a different pattern of effects on platelet behaviour. None of them affected the platelet response to noradrenaline, but Figure 4 shows that ethinyl oestradiol, stilboestrol, and mestranol, $1.0 \mathrm{mg} /$ day, caused platelet sensitivity to become abnormal, as already defined. The only natural oestrogen preparation, Premarin, had no detectable effect on the platelets, even when $10 \mathrm{mg}$ was administered daily for two weeks and the subject was complaining of marked side effects.

The administration of synthetic oestrogens to

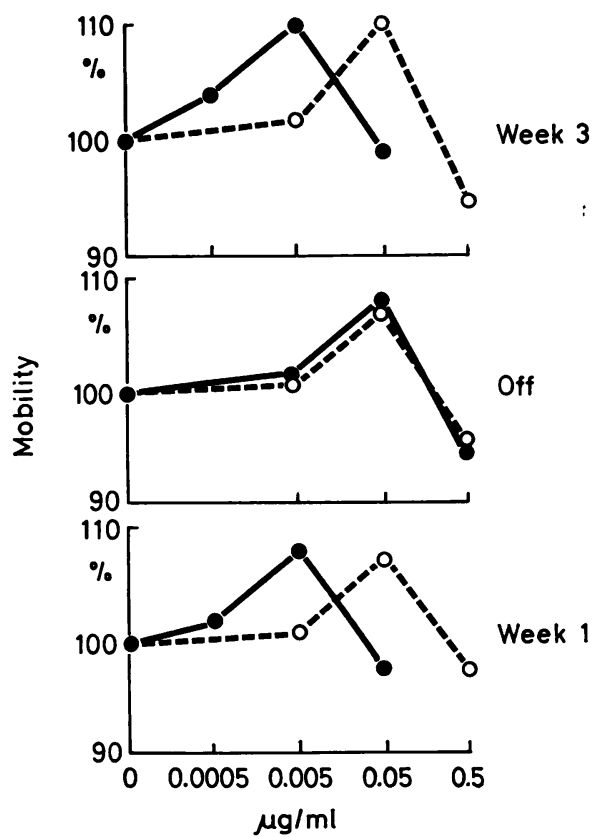

Fig. 5. Effects of adenosine diphosphate and noradrenaline on platelet mobility during and between adjacent cycles of oral contraceptive administration $(n=4)$. (Filled circles $=$ ADP; open circles $=$ noradrenaline.)

normal subjects thus makes their platelets behave like those of patients with arterial disease: there is abnormal sensitivity to ADP but normal sensitivity to noradrenaline.

\section{Effect of Oral Contraceptives in Women}

We found that oral contraceptive agents containing oestrogens had the same effect on the electrophoretic response of the platelets of the women we studied as the synthetic oestrogens had on the platelets of the men: the details of our observations have been published elsewhere (Bolton, Hampton, and Mitchell, 1968). In all the women platelet sensitivity to ADP became abnormal during the treatment phase of the cycle, but it returned to normal during the weeks between cycles. At no time was the response to noradrenaline affected. Figure 5 shows the effects of ADP and noradrenaline in four subjects studied during the third week of treatment, at the end of the subsequent week when they were untreated, and at the end of the first week of the next cycle. The 'sequential' contraceptive preparations had the same effect on the platelets as the combined oestrogen-progestogen preparations.

Two subjects were studied on at least four occasions during a single treatment cycle and Figure 6 shows the results obtained in one of these. Before beginning treatment this subject's platelet sensitivity was normal but by day 4 of 

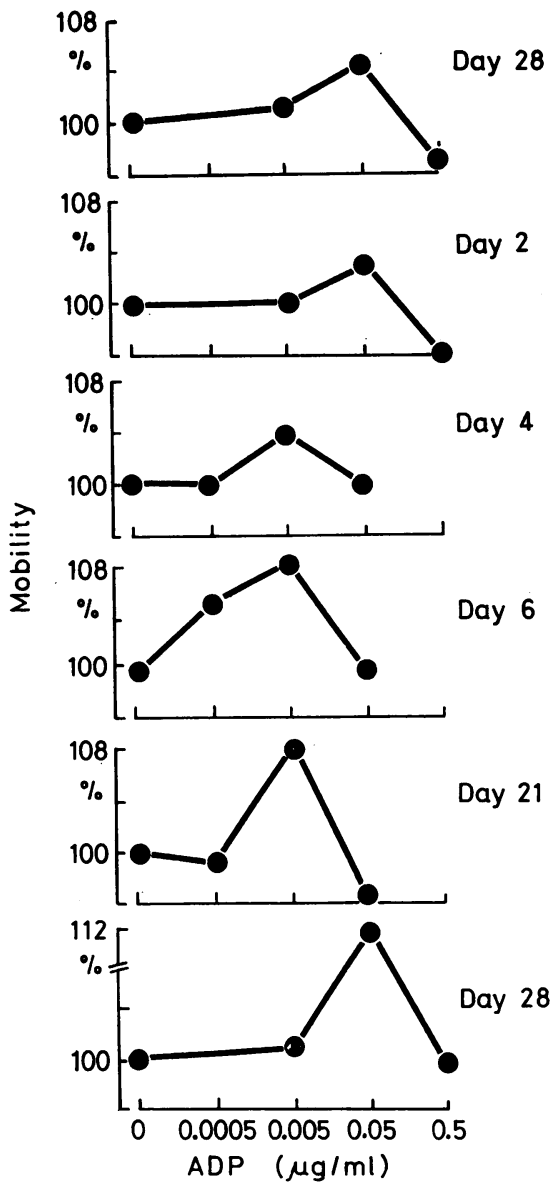

Fig. 6

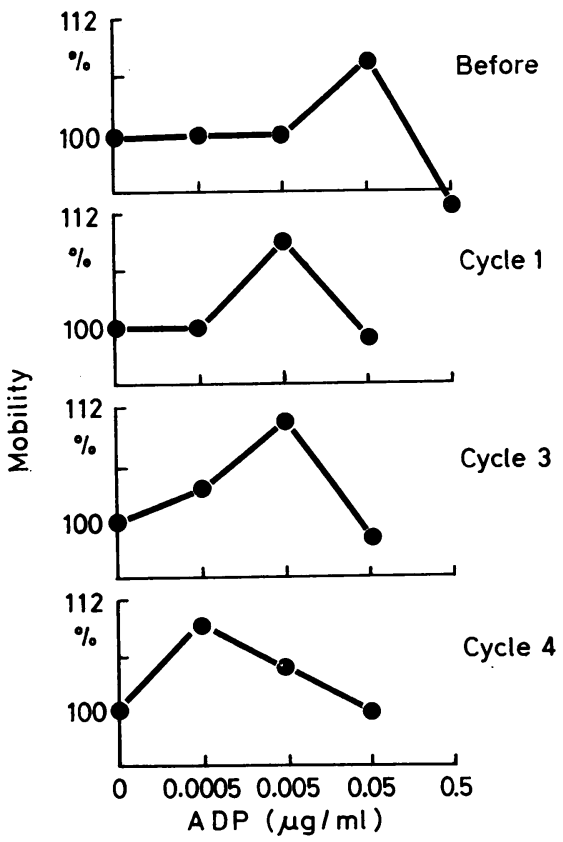

Fig. 7
Fig. 6. Platelet electrophoretic response to adenosine diphosphate during a single cycle of oral contraceptiveo administration.

Fig. 7. Effect of adenosine diphosphate on platelet mobility during the first four cycles of oral contraceptive administration in one subject.

the treatment cycle it had become abnormal From day 6 to day 21 the platelet response to ADP remained virtually unchanged.

In 10 of 11 subjects the contraceptive agents induced a ten-fold increase in platelet sensitivit to ADP, so that the maximum recorded platelex mobility was induced by $0.005 \mu \mathrm{g} / \mathrm{ml}$. In th $\overrightarrow{\mathrm{S}_{0}}$ remaining subject a similar increase occurred during the first three cycles of treatment bu during the fourth cycle her platelet sensitivitक increased by a further factor of 10 , the maximum. mobility being induced by $0.0005 \mu \mathrm{g} / \mathrm{ml}$ of ADP (Figure 7).

The platelets of the three subjects treated with chlormadinone (two of whom took $0.5 \mathrm{mg}$ daily, and the other $1.0 \mathrm{mg}$ daily) for two weekso remained normal throughout.

\section{The Cause of Abnormal Platelet Sensitivity ADP}

Having shown that synthetic oestrogens induces an abnormality in platelet electrophoretic be haviour resembling that seen in patients wi arterial disease, we next carried out a series of experiments similar to those we used to elucidafe the nature of the plasma abnormality in arteris disease (Bolton et al, 1967). The details of these experiments have been reported elsewhere (Boltom et al, 1968).

The electrophoretic technique is particularß useful for investigating plasma factors influencin platelet behaviour because 'cross-over' exper: ments between normal and abnormal platelets and plasma are easily performed. To measure platelet mobility platelet-rich plasma is diluted 1 in 10 with platelet-poor plasma and the latter can be prepared from the same blood sample the platelet-rich plasma, or from a completedy separate one. Thus using our arbitrary definitions of 'normal' and 'abnormal' platelet behaviour, +9 th is possible to use normal platelets as indicators of the presence of plasma factors that change the response of platelets to adenosine diphosphate. $\stackrel{\circ}{\circ}$

For these experiments, platelet-rich plasma wis prepared from healthy male subjects and the response of the platelets to ADP was shown to normal. When such platelet-rich plasma wo s diluted and incubated with platelet-poor plasina from a woman taking oral contraceptives whoşe 
own platelets behaved abnormally, the response of the 'normal' platelets became 'abnormal'. Platelet-poor plasma from a woman taking oral contraceptives therefore can be called 'abnormal', for it contains some factor that sensitizes normal platelets to adenosine diphosphate.

As with the plasma system that affects platelets in patients with arterial disease, we found that two components were necessary for plasma from a woman taking oral contraceptives to influence normal platelets. One of these components was stable at room temperature, and was only present in the abnormal platelet-poor plasma. The other component was unstable unless the plasma was stored at $4^{\circ} \mathrm{C}$, but it was present in both normal and abnormal platelet-poor plasma. It therefore seemed that, as with the plasma of patients with arterial disease, it was the stable component that was of more immediate interest. Filtration of abnormal platelet-poor plasma through Sephadex G200 columns showed that the stable factor activity was limited to the fractions containing substances of molecular weight greater than 200,000 . This again was similar to the 'stable factor' of the plasma system in arterial disease, and it therefore seemed likely that an abnormal lipoprotein was present in the plasma of women taking oral contraceptives. Ultracentrifugation confirmed that the stable factor in the plasma of such subjects was a lipoprotein of density between 1.006 and 1.063. Extraction with chloroformmethanol and subsequent separation of the phospholipids by thin-layer chromatography showed that the active part of these lipoproteins was their lecithin.

The administration of oral contraceptives containing oestrogens therefore causes some change in the lecithin of the plasma low-density lipoproteins, and this change is responsible for the abnormalities in platelet electrophoretic behaviour that I have described.

\section{Discussion}

At present we can only conjecture whether abnormalities in the platelet behaviour of women taking oral contraceptives are related to the small increase in the risk of vascular disease. Since the same abnormality of platelet electrophoretic behaviour (apparently due to the same abnormality of plasma lipids) seems to be present in women taking oral contraceptives and in patients with arterial disease, and since this abnormality has not been demonstrated in any other pathological conditions except some hyperlipidaemias, it is tempting to suppose that it is related to vascular disease.

We have no evidence that oral contraceptives induce an intrinsic platelet defect: it seems likely that the platelets remain normal, but that their behaviour is modified by an abnormality in the plasma lipids. The important lipid fraction seems to be the lecithin of the low-density lipoproteins: how this differs from normal we do not know, and the abnormality could have either a chemical or a physical basis. The half-life of the plasma low-density lipoproteins is about three days (Moser and Emerson, 1955; Walton, Scott, Jones, Fletcher, and Whitehead, 1963) and, since the abnormal behaviour in platelets is established after about five days, it seems likely that the basic effect of synthetic oestrogens is to induce the liver to produce an abnormal lipoprotein.

The role of the labile component of the plasma system also remains to be clarified : circumstantial evidence suggests that at least part of it is an enzyme that converts lecithin to lysolecithin, and that lysolecithin is ultimately responsible for the increased platelet sensitivity to ADP (Hampton and Bolton, 1969).

One of the most striking features of the changes in platelet behaviour induced by oestrogens is their marked dependence on the stage of the treatment cycle. Platelets become abnormal after four or five days of treatment, and revert to normal in a similar period when treatment is discontinued. Any metabolic study of the action of oral contraceptives must take account of the possibility of rapid changes, and observations must be made at defined points in the treatment cycle.

It is not clear from our limited study whether a platelet function test could be used to identify the women particularly at risk from oral contraceptives. The different effects of oestrogens on platelet adhesiveness, aggregation, and electrophoretic mobility response emphasize that until we know which (if any) platelet function tests are relevant to thrombosis, we cannot draw clinically useful conclusions from them. Measurement of the electrophoretic mobility response appears to give the most information about individual subjects, but unfortunately the technique is tedious and time-consuming. At present it seems more important to concentrate on establishing the nature of the lipoprotein abnormality induced by oral contraceptives than to study the platelets of large groups of women.

The observations that I have described have two important implications. First, oral contraceptives have many effects other than the suppression of ovulation. Second, contraceptive preparations that do not contain synthetic oestrogens may not be associated with an increased risk of vascular disease.

These experiments were performed jointly with Dr C. J. Bolton, Dr R. Elkeles, and Professor J. R. A. Mitchell. We are grateful to G. D. Searle and Co., and to their medical director, Dr G. R. Venning, for providing materials and apparatus for these and other studies. We are indebted to Dr G. A. Christie of Syntex Pharmaceuticals Ltd for supplies of chlormadinone. 


\section{References}

Bolton, C. H., Hampton, J. R., and Mitchell, J. R. A. (1967). Nature of the transferable factor which causes abnormal platelet behaviour in vascular disease. Lancet, 2, 11011105 .

Bolton, C. H., Hampton, J. R., and Mitchell, J. R. A. (1968) Effect of oral contraceptive agents on platelets and plasma phospholipids. Lancet, 1, 1336-1341.

Born, G. V. R. (1962). Aggregation of blood platelets by adenosine diphosphate and its reversal. Nature (Lond.), 194, 927-929.

Daniel, D. G., Campbell, H., and Turnbull, A. C. (1967). Puerperal thromboembolism and the suppression of lactation. Lancet, 2, 287-289.

Elkeles, R. S., Hampton, J. R., and Mitchell, J. R. A. (1968) Effect of oestrogens on human platelet behaviour. Lancet, 315-318.

Hampton, J. R., and Bolton, C. J. (1969). Effect of phospholipids on platelet electrophoret ic mobility. J. Atheroscler. Res., 9, 131-139.

Hampton, J. R., and Mitchell, J. R. A. (1966a). Effect of aggregating agents on the electrophoretic mobility of human blood platelets. Brit. med. J., 1, 1074-1077.

Hampton, J. R., and Mitchell, J. R. A. (1966b). Abnormalities in platelet behaviour in acute illnesses. Brit. med. J., 1, 1078-1080.

Hampton, J. R., and Mitchell, J. R. A. (1966c). A transferable factor causing abnormal platelet behaviour in arterial disease. Lancet, 2, 764-768.

Inman, W. H. W., and Vessey, M. P. (1968). Investigation of deaths from pulmonary, coronary, and cerebral thrombosis and embolism in women of child-bearing age. Brit. med. J., 2, 193-199,

Kannell, W. B., Dawber, T. R., Kagan, A., Rivotskie, N., and Stokes, J., III (1961). Factors of risk in the development of coronary heart disease: six year follow-up experience. The Framingham Study. Ann. intern. Med., 55, 33-50.

Moser, H. W., and Emerson, K. (1955). Estimation of the phospholipid phosphorus turnover time in man. J. clin. Invest., 34, 1286-1296.
Vessey, M. P., and Doll, R. (1968). Investigation of relatim between use of oral contraceptives and thromboembolif disease. Brit. med. J., 2, 199-205.

Veterans Administration (1967). Treatment and survival of tients with cancer of the prostate. Report of the Co-ope tive Urological Research Group. Surg. Gynec. Obstet., 124, 1011-1017.

Walton, K. W., Scott, P. J., Jones, J. V., Fletcher, R. F., a Whitehead, T. (1963). Studies on low-density lipeprotein turnover in relation to Atromid therapy. Atheroscler. Res., 3, 396-414.

Wright, H. P. (1942). Changes in the adhesiveness of bloged platelets following parturition and surgical operatio J. Path. Bact., 54, 461-468. 